http://jmscr.igmpublication.org/home/

ISSN (e)-2347-176x ISSN (p) 2455-0450

crossref DOI: https://dx.doi.org/10.18535/jmscr/v7i10.33

Journal Of Medical Science And Clinical Research

\title{
Six Sigma: Tool for Quality and Cost Effectiveness
}

\author{
Authors
}

\section{Dr Chandana G, Dr Kavita P Rasalkar*, K Nagaraju}

Department of Biochemistry, PES Institute of Medical Science and Research, Kuppam

*Corresponding Author

Dr Kavita P Rasalkar

Department of Biochemistry, PES Institute of Medical Science and Research, Kuppam

\begin{abstract}
Introduction: The Six Sigma plan measures the degree to which any process deviates from its goal. Sixsigma is a metric that quantifies the performance of processes as a rate of Defects-Per-Million Opportunities, (DPM, or DPMO). Sigma works on a quality principle of DMAIC which stands for Define, Measure, Analyse, Improve and Control.

Aims and Objectives: To evaluate our laboratory performance from Jan 2019 to June 2019 by estimating six-sigma.

Methodology: We evaluated sigma metric for 35 parameters run in our auto analyzers from Jan 2019 to June 2019 (6 months). Coefficient of variation which represents lab precision and BIAS which represents accuracy is calculated by using standard formulas. Later average six sigma was calculated from Jan 2019 to June 2019. Average Total error also was calculated for 6 months. To know about the goals for improvement in our laboratory after estimating six sigma we calculated quality goal index.

Results and Discussion: Out of 35 parameters, we observed > 3 sigma metrics for 19 parameters of all levels. Parameters (T3, T4, TSH, Troponin I) and Gem Premier Instrument (pO2, pCO2) failed to show > 3 six sigma. The QGI showed that the problem lied with inaccuracy with these parameters. Sigma $\geq 6$ is practically impossible to achieve. Total Protein and albumin has failed to achieve > 3 sigma metrics and imprecision problem lied with these parameters. Highest sigma metric was observed in $\mathrm{pH}$, triglyceride, and lipase.

Conclusion: Our laboratory has performed considerably well after assessing through the sigma metric. Application of six sigma in laboratory can be a considerable indicator for quality and reducing number of IQC runs which can be cost effective. The drawback of not achieving standard world class six sigma all the time is still existing in healthcare. However quality reports with cost effectiveness should always be a dictum as a health care professional.

Keywords: Six Sigma, Coefficient of Variation, Total Allowable Error, Quality Goal Index.
\end{abstract}

\section{Introduction}

Clinical laboratories are playing very important role in health care services by assisting clinicians to come to a conclusion on screening, diagnosis, prognosis and monitoring diseases. Laboratory medicine is fast evolving with high end automation for faster and accurate patient results. Hence quality, efficiency and cost effectiveness in laboratory results revolves around total quality management. Total Quality management includes 
quality planning, quality laboratory processes, quality control (QC), and quality assessment (QA) and quality improvement.

The Laboratory system has recently adopted six sigma apart from the routine internal quality controls and EQAS (External quality assessment services) to assess the laboratory performance.

The six sigma was first invented by the Motorola Company in 1995 for quality production in the industry and GE Company implemented the same in their industry. Health care providers adopted sigma metrics for better health care services only recently. Clinical services can also use sigma metrics for applying quality principles, but laboratory services use this technology more intensively than other medical services. Even among all the clinical laboratories the sigma metrics cannot be applied to all disciplines. Since Clinical Biochemistry and Haematology use automation, we can use the sigma metrics readily and easily than the anatomical pathology ${ }^{(1)}$.

The Six Sigma plan measures the degree to which any process deviates from its goal.

Sigma works on a quality principle of DMAIC which stands for Define, Measure, Analyse, Improve and $\mathrm{Control}^{(2)}$.

The sigma is measured in the analytical phase of total testing process. The sigma value shows how often errors or defect are likely to occur and therefore it is a metric that quantifies the performance of processes as a rate of defects-PerMillion Opportunities, (DPM, or DPMO). 1 sigma $(\sigma)$ corresponds to $6,90,000$ defects or errors per million reports, 2 sigma corresponds to $3,08,000$ errors per million reports, 3 sigma corresponds to 66,800 errors per million reports, 4 sigma corresponds to 6,210 errors per million reports, 5 sigma corresponds to 230 errors per million reports and 6 sigma corresponds to 3.4 errors per million reports. $6 \sigma$ is the best, or 'world class quality,' performance level ${ }^{(3)(4)}$.

\section{Bench Markings of Sigma Metrics}

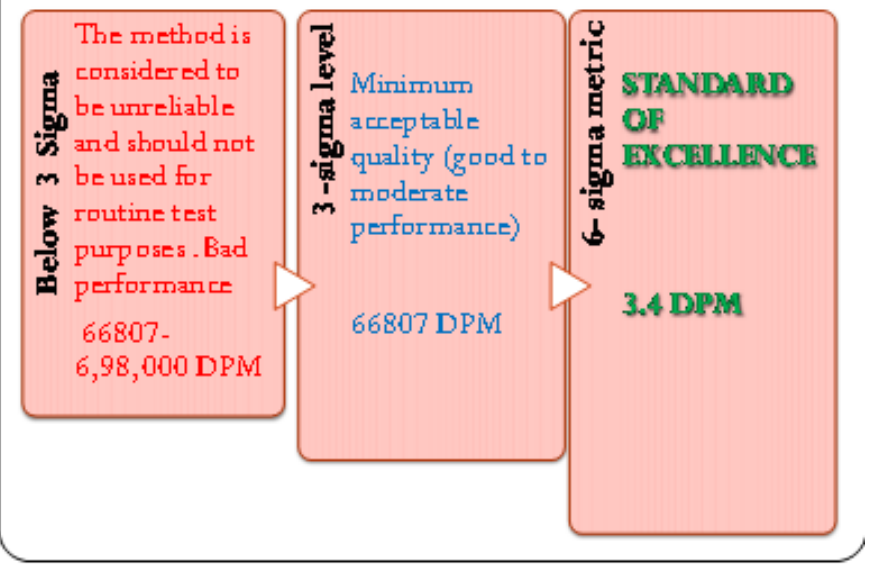

We cannot reach "world class quality" six sigma in the health care sector for many situations. Even otherwise we have to make effort to decrease the number of defects to bring it to zero defects.

\section{Aims and Objectives}

To evaluate our laboratory performance from Jan 2019 to June 2019 by estimating six sigma.

\section{Materials and Methods}

We evaluated sigma metric for 35 parameters run in our auto analyzers from Jan 2019 to June 2019 (6 months).Clinical chemistry were analyzed in Vitros FS 5.1 integrated system and Vitros 250 (ortho clinical Diagnostic) by reflectance spectrophotometry. HbA1C was measured in Biorad D10 instrument by HPLC method. The ABG analysis was measured by the Gem Premier 2000 through Ion Selective electrode method .Thyroid function tests (T3, T4, TSH,fT3 and fT4), Troponin I were analyzed by Vidas and Mini Vidas by ELFA (Enzyme linked fluorescent assay)

Steps for calculating Sigma Metric

Step 1: The Internal QC (Quality control) is run on daily basis.

IQC design in our lab as per NABL ISO 15189 guidelines

Lot numbers: (Jan 2019 -June 2019)

1. Lypochek: 26440

2. Cardiac Markers: $2^{\text {nd }}$ Party control Performance verifier PV1 (Y6491), PV2 (A6493)

3. Immunoassay: 40350 
4. Diabetic Control (HbA1C): 33970

5. Blood gas control: 29520 (Jan 2019 - March 2019) and 29540 (April 2019 to June 2019)

6. Troponin I: Variable lots (Second party controls)

For analytes load >75 investigations like glucose, urea ,creatinine ,total bilirubin (TB), Direct bilirubin (DB), electrolytes (Sodium, Potassium .Chloride)we run 2 levels of QC (Level 1 and Level 2) two times a day .

- For analytes load $<75$ investigations like Lipid profile (Triglyceride, Total cholesterol, High Density Lipoprotein)), minerals (calcium, phosphorus and magnesium), Enzymes (Amylase, Lipase, AST, ALT, ALP, CK, CKMB, Cholinesterase, GGT), Arterial blood gas analysis (pO2, $\mathrm{pCO} 2, \mathrm{pH}$, glucose, lactate) alternatively level 1 and 2 of $\mathrm{QC}$ is run every day.

○ 3 levels (Level 1, Level 2, and Level 3) are run for $A B G$ analysis and Thyroid hormones. All other parameters only 2 levels (Level 1 and Level 2) are available.

The QC was entered manually in the software with LJ (Levy Jennings chart). West gard rules are applied to identify random and systematic errors .Corrective and preventive actions are taken and documented. The lab mean and SD (Standard deviation) was calculated automatically by the software.

CV (Coefficient of variation) represents the lab precision. Precision has been defined as the closeness of agreement between independent results of measurements obtained under stipulated conditions. CV was calculated every month by the formula (SD/Mean)*100.

Step 2: We participate in the External Quality Assurance Services (EQAS) programs from Biorad EQAS QC Cycle 17 (Sample 7, 8, 9, 10, 11 , 12) were run for Jan 2019- June 2019 (6 months) which was run every month for all the biochemical and immunoassay analytes. High sensitive Troponin I was covered under cardiac marker EQAS. Bias provides the lab accuracy (Trueness or accuracy is defined as closeness of agreement between the average value obtained from a large series of results of measurements and the true value).BIAS is calculated by using the formula

BIAS = (Laboratory Result - Reference Value or True Value) /Reference Value or True Value.

Step 3: Total Error Allowable (TEa) is the maximum error allowed in a laboratory for a particular measurement procedure. This includes variations in all phases of testing process. Total allowable error values for each analyte was selected by various approaches and strategies

$>$ Based on biological variations

$>$ Based on Professional recommendations by International expert groups (CLIA, RICOS,RILIBAK $(12,13,14)$

$>$ EQA Published data

TEa (Total Allowable error) in our study was taken from the RICOS guidelines (Dr. Carmen Ricos and colleagues) (5, 13). For analytes like pCO2 where the TEa was not available we used RCPA guidelines ${ }^{(6)}$

Sigma metric was calculated using the formula (TEa - BIAS) / CV

Errors in analytical phases have two main components: random and systematic errors.

Using these two components, we can calculated the total error of a test as

$$
\mathrm{TE}=\mathrm{Bias}+1.65 \mathrm{CV}
$$

The Sigma metric, TE was calculated for each month from Jan 2019 to June 2019. Average Sigma metric was taken for assessing our lab performance.

After determining our sigma metrics, we needed to take some corrective and preventive action. To identify this we calculated quality goal index (QGI). Quality goal index was used to find out whether deviation was observed in bias or precision. This helps us to know whether the analytes showed imprecision or inaccuracy or both. The formula to calculate QGI is (Bias /1.5)*CV\%. 
Criteria used for interpreting QGI when test applications fall short of six sigma $<3$ quality. ${ }^{(7,}$ 8)

\begin{tabular}{|l|l|}
\hline QGI & Problem \\
\hline$<0.8$ & Imprecision \\
\hline $0.8-1.2$ & Imprecision and Inaccuracy \\
\hline$>1.2$ & Inaccuracy \\
\hline
\end{tabular}

\section{Results}

Average CV (Level 1 and Level 2) and average BIAS was taken from Jan 2019 to June 2019 for the analytes measured in our analyzers. Colours highlighted in table as follows

Red: $<3$ sigma

Green: Highest sigma

Pink: Lowest total error

Blue: Highest total error The parameters not highlighted have $>3$ sigma levels

Table 1: Sigma metrics of Vitros 250 analyzer (Level 1 and Level 2)

\begin{tabular}{|c|c|c|c|c|c|c|c|c|c|}
\hline SI.No & $\begin{array}{c}\text { Parameters } \\
\text { Vitros 250 }\end{array}$ & $\begin{array}{l}\text { Avg } \\
\text { CV } \\
\text { L1 }\end{array}$ & $\begin{array}{l}\text { Avg } \\
\text { CV } \\
\text { L2 }\end{array}$ & $\begin{array}{c}\text { Avg } \\
\text { BIAS }\end{array}$ & $\begin{array}{c}\text { TEa } \\
\text { (RICOS) }\end{array}$ & $\begin{array}{l}\text { Six Sigma } \\
\text { (L1) }\end{array}$ & $\begin{array}{l}\text { Six Sigma } \\
\text { (L2) }\end{array}$ & $\begin{array}{c}\text { Avg } \\
\text { (Total } \\
\text { Error) } \\
\text { L1 }\end{array}$ & $\begin{array}{c}\text { Avg } \\
\text { (Total } \\
\text { Error) } \\
\text { L2 }\end{array}$ \\
\hline 1 & Glucose & 2.40 & 2.25 & -0.15 & 5.5 & 2.36 & 2.51 & 5.54 & 5.25 \\
\hline 2 & Sodium & 1.50 & 1.02 & -0.17 & 0.73 & 0.60 & 0.88 & 2.44 & 1.66 \\
\hline 3 & Potassium & 1.58 & 1.20 & -0.11 & 5.61 & 3.62 & 4.77 & 2.60 & 1.98 \\
\hline 4 & Chloride & 0.84 & 0.94 & -0.14 & 1.5 & 1.95 & 1.74 & 1.37 & 1.53 \\
\hline 5 & Triglyceride & 1.93 & 2.20 & -0.13 & 25.99 & 13.51 & 11.87 & 3.79 & 4.31 \\
\hline 6 & Total Cholesterol & 2.92 & 3.13 & -0.14 & 9.01 & 3.14 & 2.92 & 5.68 & 6.11 \\
\hline 7 & HDL & 3.47 & 4.17 & $\begin{array}{l}-1.09 \\
\end{array}$ & 11.63 & 3.67 & 3.05 & 6.81 & 8.18 \\
\hline 8 & Bilirubin-Total & 5.83 & 3.38 & -1.11 & 26.94 & 4.81 & 8.29 & 11.43 & 6.63 \\
\hline 9 & Total Protein & 2.23 & 2.38 & -0.05 & 3.63 & 1.65 & 1.54 & 4.38 & 4.68 \\
\hline 10 & Albumin & 2.68 & 2.52 & 2.15 & 4.07 & 0.72 & 0.76 & 5.25 & 4.93 \\
\hline 11 & AST & 2.92 & 3.42 & -0.11 & 16.69 & 5.76 & 4.92 & 5.70 & 6.68 \\
\hline 12 & ALT & 6.85 & 3.33 & -0.13 & 27.48 & 4.03 & 8.28 & 13.44 & 6.55 \\
\hline 13 & ALKP & 3.23 & 2.73 & -0.85 & 15.4 & 5.03 & 5.95 & 6.32 & 5.34 \\
\hline 14 & Urea & 3.45 & 3.18 & -0.68 & 15.55 & 4.71 & 5.10 & 6.75 & 6.23 \\
\hline 15 & Creatinine & 2.40 & 3.05 & -0.04 & 8.87 & 3.71 & 2.92 & 4.67 & 5.95 \\
\hline
\end{tabular}

The highest sigma was seen in parameter triglyceride (Level 1 and Level 2). The total error was lowest for chloride parameter. The total error was highest in AST (Level 2) and ALT (Level 1).

Table 2: Sigma metrics of Vitros FS 5.1 analyzer (Level 1 and Level 2)

\begin{tabular}{|c|c|c|c|c|c|c|c|c|c|}
\hline SI.No & $\begin{array}{c}\text { Parameters } \\
\text { Vitros FS5.1 }\end{array}$ & $\begin{array}{l}\text { Avg } \\
\text { CV } \\
\text { L1 }\end{array}$ & $\begin{array}{l}\text { Avg } \\
\text { CV } \\
\text { L2 }\end{array}$ & $\begin{array}{c}\text { Avg } \\
\text { BIAS }\end{array}$ & $\begin{array}{c}\text { TEa } \\
\text { (RICOS) }\end{array}$ & $\begin{array}{c}\text { Six Sigma } \\
\text { (L1) }\end{array}$ & $\begin{array}{c}\text { Six } \\
\text { Sigma } \\
\text { (L2) }\end{array}$ & $\begin{array}{c}\text { Avg } \\
\text { (Total } \\
\text { Error) } \\
\text { L1 }\end{array}$ & $\begin{array}{c}\text { Avg } \\
\text { (Total } \\
\text { Error) } \\
\text { L2 }\end{array}$ \\
\hline 1 & Glucose & 1.57 & 1.62 & -0.34495 & 5.5 & 3.73 & 3.62 & 3.08 & 3.18 \\
\hline 2 & Sodium & 1.63 & 1.50 & $\begin{array}{l}-0.3321 \\
\end{array}$ & 0.73 & 0.65 & 0.71 & 3.19 & 2.93 \\
\hline 3 & Potassium & 1.65 & 1.82 & -0.34162 & 5.61 & 3.61 & 3.28 & 3.25 & 3.58 \\
\hline 4 & Chloride & 1.67 & 1.73 & -0.33082 & 1.5 & 1.10 & 1.06 & 3.26 & 3.39 \\
\hline 5 & Triglyceride & 1.58 & 2.12 & -0.33932 & 25.99 & 16.63 & 12.44 & 3.09 & 4.14 \\
\hline 6 & Total Cholesterol & 1.73 & 2.65 & -0.31836 & 9.01 & 5.38 & 3.52 & 3.37 & 5.17 \\
\hline 7 & HDL & 2.82 & 2.68 & -0.33674 & 11.63 & 4.25 & 4.46 & 5.53 & 5.27 \\
\hline 8 & Bilirubin-Total & 4.87 & 3.23 & -0.33703 & 26.94 & 5.60 & 8.44 & 9.55 & 6.35 \\
\hline 9 & Total Protein & 1.80 & 2.10 & -0.33575 & 3.63 & 2.20 & 1.89 & 3.53 & 4.12 \\
\hline 10 & Albumin & 1.88 & 1.98 & -0.33522 & 4.07 & 2.34 & 2.22 & 3.70 & 3.89 \\
\hline 11 & AST & 2.72 & 2.97 & -0.33746 & 16.69 & 6.27 & 5.74 & 5.32 & 5.81 \\
\hline 12 & ALT & 7.87 & 2.98 & -0.34025 & 27.48 & 3.54 & 9.33 & 15.43 & 5.86 \\
\hline 13 & ALKP & 2.15 & 2.28 & -0.35039 & 15.4 & 7.33 & 6.90 & 4.21 & 4.47 \\
\hline
\end{tabular}




\begin{tabular}{|l|c|c|c|c|c|c|c|c|c|}
\hline 14 & GGT & 2.13 & 1.67 & -0.32371 & 22.11 & 10.52 & 13.46 & 4.14 & 3.23 \\
\hline 15 & Urea & 2.75 & 2.43 & -0.3184 & 15.55 & 5.77 & 6.52 & 5.41 & 4.79 \\
\hline 16 & Creatinine & 2.35 & 2.47 & -0.31648 & 8.87 & 3.91 & 3.72 & 4.62 & 4.85 \\
\hline 17 & Uric acid & 2.43 & 2.42 & -0.3412 & 11.97 & 5.06 & 5.09 & 4.76 & 4.72 \\
\hline 18 & Total Calcium & 1.53 & 1.75 & -0.32016 & 2.55 & 1.87 & 1.64 & 2.99 & 3.41 \\
\hline 19 & Phosphorus & 1.52 & 2.35 & -0.31144 & 10.11 & 6.87 & 4.43 & 2.97 & 4.61 \\
\hline 20 & Magnesium & 2.22 & 2.07 & -0.33302 & 4.8 & 2.32 & 2.48 & 4.33 & 4.04 \\
\hline 21 & Amylase & 6.52 & 2.32 & -0.31765 & 17.7 & 2.76 & 7.78 & 12.78 & 4.55 \\
\hline 22 & Lipase & 1.75 & 1.95 & -0.32425 & 37.88 & 21.83 & 19.59 & 3.43 & 3.82 \\
\hline 23 & Cholinesterase & 3.95 & 5.37 & -0.32309 & 9.8 & 2.56 & 1.89 & 7.70 & 10.47 \\
\hline 24 & LDH & 4.70 & 3.43 & -0.3068 & 11.4 & 2.49 & 3.41 & 9.18 & 6.70 \\
\hline
\end{tabular}

The highest sigma was seen in parameter Lipase (L1 and L2). The total error was lowest for sodium (L2) and phosphorus (L1) parameter. The

Table 3: Sigma metrics for other instrument parameters(Gem Premier-ABG, Biomeriex Vidas-TFT and H S Troponin I, Biorad D10-HbA1c )

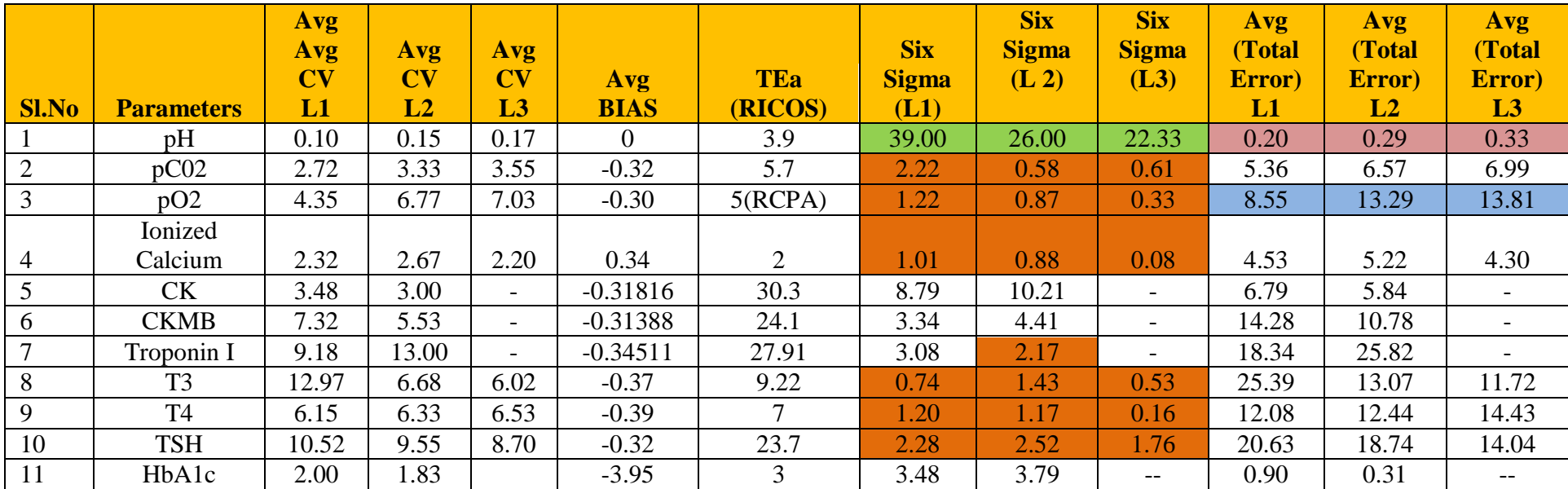

Among the $\mathrm{ABG}$ analysis was only $\mathrm{pH}$ had a highest sigma level in all levels therefore the total errors in all levels with $\mathrm{pH}$ was low. The highest total errors are seen in pCO2 (all levels). Our cardiac markers were having satisfactory sigma total error was highest in ALT (L1) and Cholinesterase (L2). 
Table 5: Quality goal index of parameters measured in Vitros FS 5.1

\begin{tabular}{|l|c|c|c|c|}
\hline Sl.No & Levels & Analytes & QGI & Problem \\
\hline \multirow{3}{*}{1} & Level 1 & Sodium & 0.36 & \\
& & & & Imprecision \\
\cline { 2 - 5 } & Level 2 & Sodium & 0.33 & Imprecision \\
\hline \multirow{2}{*}{3} & Level 1 & Chloride & 0.37 & Imprecision \\
\cline { 2 - 5 } & Level 2 & Chloride & 0.38 & Imprecision \\
\hline \multirow{3}{*}{4} & Level 1 & Total Protein & 0.40 & Imprecision \\
\cline { 2 - 5 } & Level 2 & Total Protein & 0.47 & Imprecision \\
\hline \multirow{3}{*}{5} & Level 1 & Albumin & 0.42 & Imprecision \\
\cline { 2 - 5 } & Level 2 & Albumin & 0.44 & Imprecision \\
\hline \multirow{2}{*}{6} & Level 1 & Total Calcium & 0.33 & Imprecision \\
\cline { 2 - 5 } & Level 2 & Total Calcium & 0.37 & Imprecision \\
\cline { 2 - 5 } & Level 1 & Magnesium & 0.49 & Imprecision \\
\hline 7 & Level 2 & Magnesium & 0.46 & Imprecision \\
\hline 8 & Level 1 & Amylase & 1.38 & Inaccuracy \\
\cline { 2 - 5 } & Level 2 & Cholinesterase & 0.85 & $\begin{array}{c}\text { Imprecision } \\
\text { and Inaccuracy }\end{array}$ \\
\hline 9 & Level 1 & LDhesterase & 1.16 & Inaccuracy \\
\hline & & LDH & 0.96 & Imprecision \\
& & & & \\
\hline
\end{tabular}

Many had imprecision problem but some parameters like cholinesterase had both imprecision and inaccuracy problem

Table 6: Quality goal index of parameters measured in Vidas, Gem Premier and Biorad D10

\begin{tabular}{|l|c|c|c|c|}
\hline SI.No & Levels & $\begin{array}{c}\text { Analytes } \\
\text { Level 1 }\end{array}$ & QGI & Problem \\
\hline \multirow{4}{*}{1} & Level 1 & T3 & 3.19 & Inaccuracy \\
\cline { 2 - 5 } & Level 2 & T3 & 1.65 & Inaccuracy \\
\cline { 2 - 5 } & Level 3 & T3 & 3.95 & Inaccuracy \\
\hline \multirow{4}{*}{2} & Level 1 & T4 & 1.57 & Inaccuracy \\
\cline { 2 - 5 } & Level 2 & T4 & 1.61 & Inaccuracy \\
\cline { 2 - 5 } & Level 3 & T4 & 5.53 & Inaccuracy \\
\hline \multirow{4}{*}{3} & Level 1 & TSH & 2.26 & Inaccuracy \\
\cline { 2 - 5 } & Level 2 & TSH & 2.06 & Inaccuracy \\
\cline { 2 - 5 } & Level 3 & TSH & 3.09 & Inaccuracy \\
\hline 4 & Level 2 & Troponin & 2.99 & Inaccuracy \\
\hline 5 & Level 1 & pC02 & 0.58 & Imprecision \\
\cline { 2 - 5 } & Level 2 & pCO2 & 0.71 & Imprecision \\
\cline { 2 - 5 } & Level 3 & pC02 & 2.20 & Inaccuracy \\
\hline 6 & Level 1 & pO2 & 0.87 & Imprecision and Inaccuracy \\
\cline { 2 - 5 } & Level 2 & PO2 & 1.35 & Inaccuracy \\
\cline { 2 - 5 } & Level 3 & pO2 & 10.25 & Inaccuracy \\
\hline 7 & Level 1 & Ionized calcium & 0.52 & Imprecision \\
\cline { 2 - 5 } & Level 2 & Ionized Calcium & 0.60 & Inaccuracy \\
\cline { 2 - 5 } & Level 3 & Ionized calcium & 2.38 & Inaccuracy \\
\hline
\end{tabular}

Thyroid profile parameters and ABG Parameters had more inaccuracy except for $\mathrm{pH}$ 
Figure 1: Distribution of parameters where the QGI (Quality goal Index) acts as an indicator for laboratory improvement if the parameters have less than 3 six sigma.

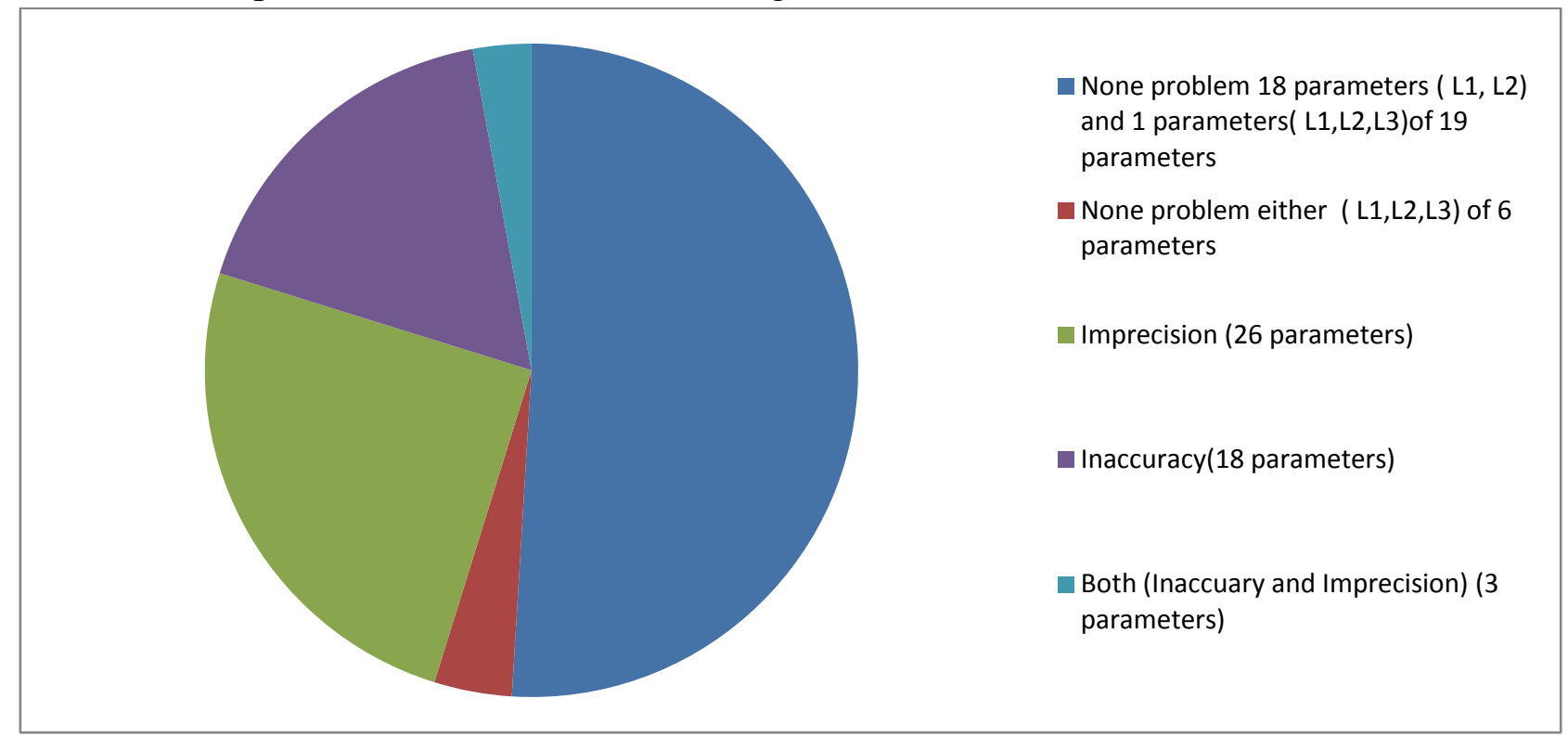

Majority of our parameters are above the 3 six sigma metrics.

\section{Discussion}

Sigma metrics is one of the tools of quality in terms of performance for any product being manufactured. The laboratory that generates products (Laboratory reports) should be of assured quality. The universal scale or bench marking, the Six Sigma for assessing the lab performance is 1 to 6. The 6 sigma shows world class: "BEST PERFORMER" and $<3$ sigma metric shows the need for improvement by interpreting Quality goal index (Problem that lies with imprecision /Inaccuracy/ Imprecision and inaccuracy).

This study analyzed our laboratory performance for 35 parameters measured in our various analyzers Vitros 250, Vitros FS 5.1, Gem Premier, Vidas and Biorad D10. The sigma metrics were analyzed for all the parameters in all levels (Vitros run analytes L1and L2, Thyroid profile (T3, T4, $\mathrm{TSH})$ and $\mathrm{ABG}(\mathrm{pH}, \mathrm{pCO} 2, \mathrm{pO} 2)$ in all the three levels L1, L2 and L3.

We observed > 3 sigma metrics for 19 parameters of all levels.

Vidas instrument parameters (T3, T4, TSH, Troponin I) and Gem Premier Instrument (pO2, pCO2) failed to show > 3 six sigma. The QGI showed that the problem lied with inaccuracy. The reason could be due to frequent reagent lot changes, improper daily maintenance of the instrument; however the preventive maintenance was done on yearly basis by the company. Frequent breakdown of instrument was also observed during Jan 2019 to June 2019. The corrective actions taken for this were to sensitize the laboratory technicians to do the daily maintenance and we have to observe the sigma metric in the next 2 months. Plan is to replace Vidas immunoassay analyzer with a fully automated one which was also suggested to the higher authority.

The electrolytes especially (Sodium and Chloride) showed <3 sigma metric in both the Vitros 250 and FS 5.1 which was much similar to the study conducted by Chakravarthy et $\mathrm{al}^{(7)}$ which also showed potassium and chloride $<3$ sigma, which was analyzed during normal conditions. Since the electrolytes like (sodium, potassium and chloride) have a total allowable error of $\leq 5 \%$ i.e. very narrow TAE. Sigma $\geq 6$ is practically impossible to achieve. The QGI for these parameters predicted imprecision. However measures can be taken to minimize the frequency of random and systematic errors by in-house training of technicians handling and running IQC in an appropriate way, as they are very sensitive 
parameters. These measures can be taken for bringing the sigma metric atleast from 3 to 6 .

Total Protein and albumin has failed to achieve > 3 sigma metrics in various studies. Bhavna sing et al, Sunil Nanda et al and Nitin Kumar et al ${ }^{(9)}$ also showed these parameters $<3$ sigma. The comparison is inevitable because these various studies have run their analytes in different instruments with different IQC and EQAS company materials. We interpreted the problem as imprecision; hence to bring up our quality again importance of training the technicians regarding IQC is inevitable.

Mostly the problem lied with imprecision with most of the parameters (General chemistry analytes). Thyroid hormones and ABG analytes showed inaccuracy problem.

\begin{tabular}{|c|c|c|c|c|c|}
\hline Sigma & Westgard Rule & Levels & Measurements & P Error Detection & P False Rejection \\
\hline 6.0 & $13.5 \mathrm{~s}$ & 2 & 1 & 0.98 & 0.01 \\
\hline 5.8 & $13.5 \mathrm{~s}$ & 2 & 1 & 0.98 & 0.00 \\
\hline 5.6 & $13 \mathrm{~s}$ & 2 & 1 & 0.97 & 0.00 \\
\hline 5.4 & $13 \mathrm{~s}$ & 2 & 1 & 0.94 & 0.00 \\
\hline 5.2 & $13 \mathrm{~s}$ & 2 & 1 & 0.91 & 0.00 \\
\hline 5.0 & $12.5 \mathrm{~s}$ & 2 & 1 & 0.96 & 0.03 \\
\hline 4.8 & $12.5 \mathrm{~s}$ & 2 & 1 & 0.93 & 0.03 \\
\hline 4.6 & $13 \mathrm{~s}$ & 2 & 1 & 0.92 & 0.01 \\
\hline 4.4 & $12.5 \mathrm{~s}$ & 2 & 1 & 0.96 & 0.04 \\
\hline 4.2 & $12.5 \mathrm{~s}$ & 2 & 1 & 0.92 & 0.04 \\
\hline 4.0 & $13 \mathrm{~s} / 22 \mathrm{~s} / \mathrm{R} 4 \mathrm{~s} / 41 \mathrm{~s}$ & 2 & 2 & 0.91 & 0.03 \\
\hline 3.8 & $13 \mathrm{~s} / 22 \mathrm{~s} / \mathrm{R} 4 \mathrm{~s} / 41 \mathrm{~s}$ & 2 & 2 & 0.86 & 0.03 \\
\hline 3.6 & $13 \mathrm{~s} / 22 \mathrm{~s} / \mathrm{R} 4 \mathrm{~s} / 41 \mathrm{~s}$ & 2 & 2 & 0.79 & 0.03 \\
\hline 3.4 & $13 \mathrm{~s} / 22 \mathrm{~s} / \mathrm{R} 4 \mathrm{~s} / 41 \mathrm{~s}$ & 2 & 2 & 0.65 & 0.03 \\
\hline 3.2 & $13 \mathrm{~s} / 22 \mathrm{~s} / \mathrm{R} 4 \mathrm{~s} / 41 \mathrm{~s}$ & 3 & 2 & 0.48 & 0.03 \\
\hline 3.0 & $13 \mathrm{~s} / 22 \mathrm{~s} / \mathrm{R} 4 \mathrm{~s} / 41 \mathrm{~s}$ & 3 & 2 & 0.36 & 0.02 \\
\hline
\end{tabular}

Based on the study findings, we could infer that six sigma is a good quality tool for assessing our laboratory performance during analytical phase. However there are certain limitations with that of six sigma application practically for few analytes especially with electrolytes analytes where the total allowable error was too low. However bias\% and imprecision ( $\mathrm{CV} \%$ ) have proven to be more reliable than sigma for assessing the performance of few analytes, but the applicable analytes have to be within the total allowable error.
The highest Sigma was obtained in Triglyceride (Vitros 250), Lipase (Vitros FS 5.1) and pH (Gem Premier) which showed lowest total error with the regard to $\mathrm{pH}$. The reason could be that the TAE of Triglyceride and lipase was very high as compared to other parameters.

However the cost effectiveness of the laboratory can be taken care by controlling/ reducing the number of daily IQC if our sigma metric has achieved 6 by limiting our application of westgard rules and number of control measurements. More number of QC and westgard rules are applied for metric <3 sigma metric. Sigma metric can also be one of our quality indicators ${ }^{(2),(10),(11)}$.

Example: QC design based on sigma metrics ${ }^{(8)}$ 
Application of six sigma in laboratory can be a considerable indicator for quality and reducing number of IQC runs which can be cost effective. The drawback of impracticality of achieving standard world class six sigma, all the time still exists in healthcare. The However quality reports with cost effectiveness should always be a dictum as a health care professional.

\section{Acknowledgement}

We thank the management of PESIMSR for giving us this opportunity to carry out the study in PESIMSR central laboratory. We thank our technical, office staff and clinical biochemist for both technical and clerical support.

\section{Conflict of interest: None}

\section{References}

1. Abdurrahman Coskun, Tamer Inal, Ibrahim Unsal and Mustafa Serteser. Six Sigma as a Quality Management Tool: Evaluation of Performance in Laboratory Medicine. www.intechopen.com

2. Study material: Workshop on Implementation of Six Sigma Metrics in Clinical Laboratories Organized by Ortho Clinical Diagnostics.

3. Sunil Kumar Nanda and Lopamudra Ray, Role. Quantitative Application of Sigma Metrics in Medical Biochemistry Journal of Clinical and Diagnostic Research. 2013 Dec, Vol-7(12): 2689-2691.

4. A Kirsch, $\mathrm{R}$ Lesiv, Ortho Clinical Diagnostics Rochester, New York; M Barba Laboratory Diagnostics Consulting, Atlanta, Georgia Comparative Study of Six Sigma Assay Performance on VITROS® Systems
5. Ricos C, Alvarez V, Cava F, Garcia-Lario JV, Hernandez A, Jimenez CV, Minchinela J, Perich C, Simon M. "Current databases on biologic variation: pros, cons and progress." Scand J Clin Lab Invest 1999;59:491-500. Database updated in 2014.

6. RCPA/AACB Chemical Pathology QAP ALPJAN11-Revision of allowable limits of performance.

7. Srinivas Chakravarthy Satish Ramanathan, Smitha S, Vijayakumar KV, Thirumalai Nallathambi, Micheal S. Phoenix in the lab: The sigma metrics during Chennai's worst disaster: Monitoring and management of the Quality Management System (QMS) IJPLM 2017;3(1):1-7

8. West Gard QC.www.westgard.com

9. Kirankumar P. Chauhan et al. Six sigma in clinical biochemistry: It matters, measure it. International Journal of Clinical Biochemistry and Research 2017; 4(3):270-274

10. Manchana Lakshman et al. Evaluation of sigma metrics in a Medical Biochemistry lab International Journal of Biomedical Research 2015; 6(03): 164-171.

11. Shilpa M agharkar. Six Sigma and quality control design. Biorad.

12. Link to access TEa: CLIA: https://www.westgard.com/clia.htm

13. Link to access TEa: RICOS Desirable : https://www.westgard.com/biodatabase1.ht $\mathrm{m}$

14. Link to access TEa: RCPA: https://www.westgard.com/rcpabiochemistry.htm 